\title{
Updated measurements of hadronic $B$ decays at CDF
}

\author{
Michael J. Morello*i \\ Scuola Normale Superiore di Pisa and INFN Sezione di Pisa \\ E-mail: michael.morellodpi.infn.it
}

The CDF experiment at the Tevatron $p \bar{p}$ collider established that extensive and detailed exploration of the $b$-quark dynamics is possible in hadron collisions, with results competitive and supplementary to those from $e^{+} e^{-}$colliders. This provides a rich, and highly rewarding program that has currently reached full maturity. In the following I report some recent results on hadronic decays: the evidence for the charmless annihilation decay mode $B_{s}^{0} \rightarrow \pi^{+} \pi^{-}$, and the first reconstruction in hadron collisions of the suppressed decays $B^{-} \rightarrow D\left(\rightarrow K^{+} \pi^{-}\right) K^{-}$and $B^{-} \rightarrow D\left(\rightarrow K^{+} \pi^{-}\right) \pi^{-}$.

The 2011 Europhysics Conference on High Energy Physics, EPS-HEP 2011,

July 21-27, 2011

Grenoble, Rhône-Alpes, France

${ }^{*}$ Speaker.
† On behalf of the CDF Collaboration. 


\section{Evidence for the charmless annihilation decay mode $B_{s}^{0} \rightarrow \pi^{+} \pi^{-}$}

Two-body non-leptonic charmless decays of $b$-hadrons are very widely studied processes in flavor physics. The variety of open channels involving similar final states provides useful experimental information to improve the accuracy of effective models of strong interaction dynamics. Some decays receive contributions from higher-order ('penguin') transitions, and are therefore sensitive to the possible presence of new physics in internal loops.

The $B_{s}^{0} \rightarrow \pi^{+} \pi^{-}$and $B^{0} \rightarrow K^{+} K^{-}$decay modes have a special status, in that all quarks in the final state are different from those in the initial state. This limits the possible diagrams that can contribute to these decay to penguin-annihilation (PA) and $W$-exchange (E) topologies. They have never been observed, and the best upper limits come from [1, 2]. These amplitudes are difficult to predict within the current phenomenological models, and are often neglected in calculations of decays where they are not the only contributors, using generic argument of smallness. These diagrams may carry different $\mathrm{CP}$-violating and $\mathrm{CP}$-conserving phases with respect to leading diagrams; the lack of knowledge of their size therefore introduces unavoidable uncertainties in the conclusions drawn from the analyses of other well-studied decays, like $B^{0} \rightarrow \pi^{+} \pi^{-}$and $B_{s}^{0} \rightarrow K^{+} K^{-}$. A measurement of branching fraction of both $B_{s}^{0} \rightarrow \pi^{+} \pi^{-}$and $B^{0} \rightarrow K^{+} K^{-}$would be particularly useful, as it allow a better determination of the strength of PA and $E$ amplitudes.
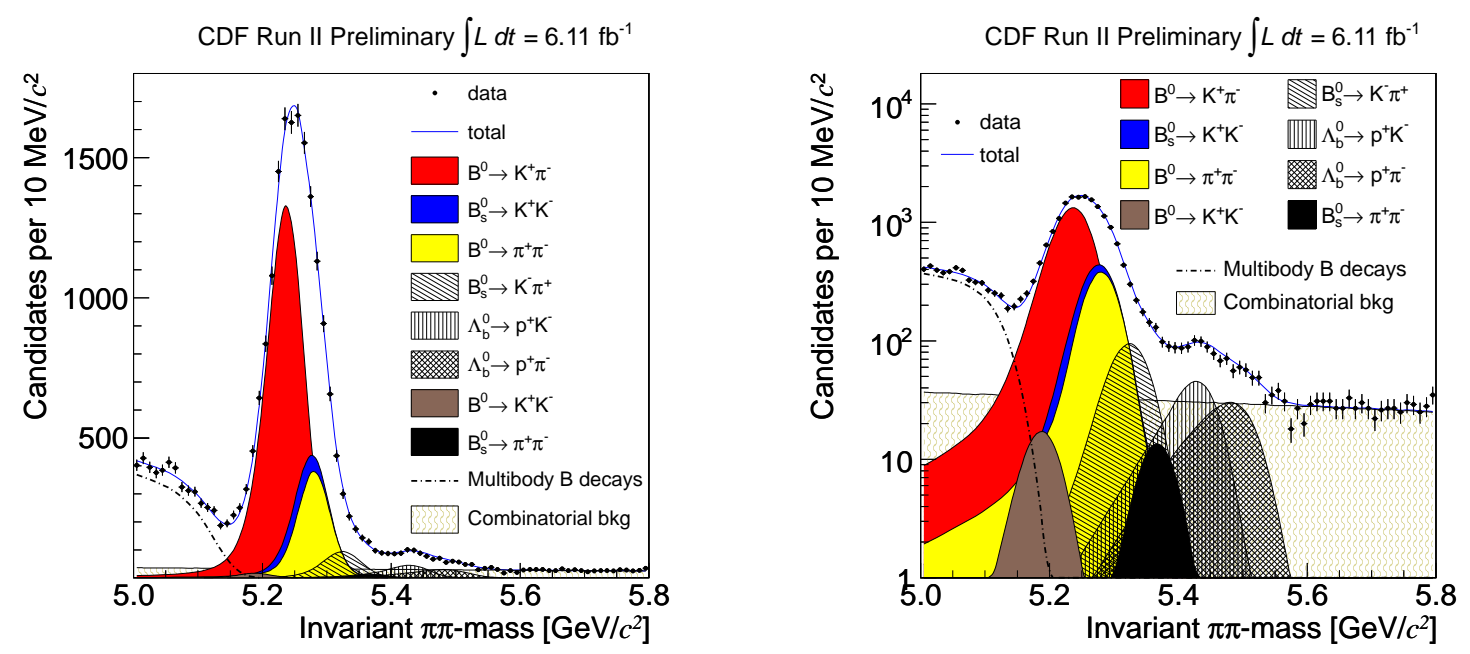

Figure 1: Invariant mass distribution of reconstructed $B_{(s)}^{0} \rightarrow h^{+} h^{\prime-}$ candidates (linear scale on the left and logarithmic scale on the right). The charged pion mass is assigned to both tracks. The sum of the fitted distributions and the individual components of signal and background are overlaid on the data distribution.

CDF recently searched for both $B_{s}^{0} \rightarrow \pi^{+} \pi^{-}$and $B^{0} \rightarrow K^{+} K^{-}$, using a data sample of $6 \mathrm{fb}^{-1}$ of integrated luminosity. An extended unbinned likelihood fit, incorporating kinematic (invariant mass and momenta) and particle identification ( $\mathrm{dE} / \mathrm{dx})$ information, is used to determine the fraction of each individual $B_{(s)}^{0} \rightarrow h^{+} h^{-}$(where $h$ is for a pion or kaon) mode in the sample. The fit projection on the invariant $\pi \pi$-mass is reported in fig. 1. A 3.7 $\sigma$ significant signal is observed for the $B_{s}^{0} \rightarrow$ $\pi^{+} \pi^{-}$mode, while a deviation at the $2 \sigma$ level from no signal hypothesis is reported for the $B^{0} \rightarrow$ $K^{+} K^{-}$. The measured branching ratios are $\mathscr{B}\left(B_{s}^{0} \rightarrow \pi^{+} \pi^{-}\right)=(0.57 \pm 0.15 \pm 0.10) \times 10^{-6}$ and 
$\mathscr{B}\left(B^{0} \rightarrow K^{+} K^{-}\right)=(0.23 \pm 0.10 \pm 0.10) \times 10^{-6}$ [阿]. A $90 \%$ confidence interval for the $B^{0} \rightarrow K^{+} K^{-}$ mode is also reported $[0.05,0.46] \times 10^{-6}\left[\right.$ [3]. The result for the $B_{s}^{0} \rightarrow \pi^{+} \pi^{-}$mode is consistent with the previous upper limit $\left(<1.2 \times 10^{-6}\right.$ at $90 \%$ C.L. $)$, based on a subsample of the current data [2] and it is in agreement with the very recent result from LHCb [4]. This agrees with the predictions in Ref. [5] within the pQCD approach. It is higher than most other predictions [6, 7, 8]. The present measurement of $\mathscr{B}\left(B^{0} \rightarrow K^{+} K^{-}\right)$supersedes the previous limit [2]. The central value

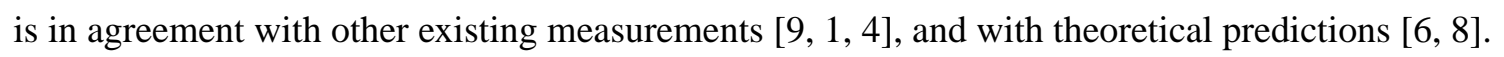

\section{2. $\gamma$ from $B \rightarrow D K$ decays}

Conventionally, $\mathrm{CP}$ violating observables are written in terms of the angles $\alpha, \beta$ and $\gamma$ of the "Unitarity Triangle", obtained from one of the unitarity conditions of the CKM matrix. While the resolution on $\alpha$ and $\beta$ reached a good level of precision, the measurement of $\gamma$ is still limited by the smallness of the branching ratios involved in the processes. Among the various methods for the $\gamma$ measurement, those which make use of the tree-level $B^{-} \rightarrow D^{0} K^{-}$decays have the smallest theoretical uncertainties. In fact $\gamma$ appears as the relative weak phase between two amplitudes, the favored $b \rightarrow c \bar{u} s$ transition of the $B^{-} \rightarrow D^{0} K^{-}$, whose amplitude is proportional to $V_{c b} V_{u s}$, and the color-suppressed $b \rightarrow u \bar{c} s$ transition of the $B^{-} \rightarrow \bar{D}^{0} K^{-}$, whose amplitude is proportional to $V_{u b} V_{c s}$. The interference between $D^{0}$ and $\bar{D}^{0}$, decaying into the same final state, leads to measurable CPviolating effects, from which $\gamma$ can be extracted. The effects can be also enhanced choosing the interfering amplitudes of the same order of magnitude. All methods require no tagging or timedependent measurements, and many of them only involve charged particles in the final state.
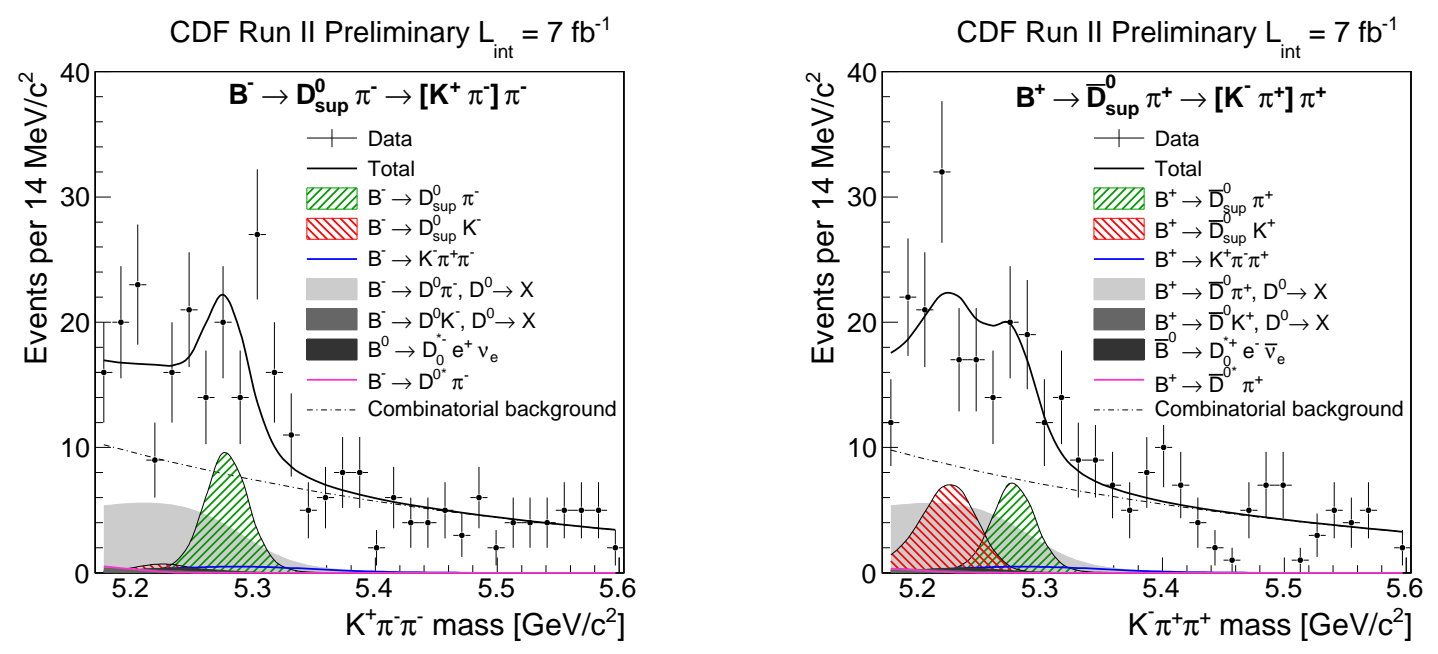

Figure 2: Invariant mass distributions of $B^{ \pm} \rightarrow D h^{ \pm}$for the suppressed mode (bottom meson on the left and antibottom on the right). The pion mass is assigned to the charged track from the B candidate decay vertex. The projections of the likelihood fit are overlaid.

In a data sample of about $7 \mathrm{fb}^{-1} \mathrm{CDF}$ reports the first reconstruction in hadron collisions of the suppressed decays $B^{-} \rightarrow D\left(\rightarrow K^{+} \pi^{-}\right) K^{-}$and $B^{-} \rightarrow D\left(\rightarrow K^{+} \pi^{-}\right) \pi^{-}$, which are the main ingredient of the the ADS method [10]. Also in this case an extended unbinned likelihood fit, 
incorporating kinematic (invariant mass) and particle identification $(\mathrm{dE} / \mathrm{dx})$ information, is used to determine the fraction of each individual modes. The fit projection on the invariant $K \pi \pi$-mass is reported in fig. 2. CDF measures the following asymmetries: $A_{A D S}(K)=-0.82 \pm 0.44$ (stat) \pm 0.09 (syst) and $A_{A D S}(\pi)=0.13 \pm 0.25$ (stat) \pm 0.02 (syst) [11], and for the ratios of doubly Cabibbo suppressed mode to flavor eigenstate CDF finds $R_{A D S}(K)=[22.0 \pm 8.6$ (stat) $\pm 2.6($ syst $)] \times 10^{-3}$ and $R_{A D S}(\pi)=[2.8 \pm 0.7$ (stat) \pm 0.4 (syst) $] \times 10^{-3}[11]$. The results are in agreement with existing measurements performed at $\Upsilon(4 \mathrm{~S})$ resonance [12] and very recently at $\mathrm{LHCb}[13]$.

\section{Conclusions}

CDF experiment at the Tevatron keeps providing excellent results in the exploration of Heavy Flavor Physics, owing to CP-symmetric initial states in $\sqrt{s}=1.96 \mathrm{TeV} p \bar{p}$ collisions, large event samples collected by well-understood detector, and mature analysis techniques. In summary, this short write-up reports on the first evidence for the charmless annihilation decay mode $B_{s}^{0} \rightarrow \pi^{+} \pi^{-}$, an updated upper limit for the $B^{0} \rightarrow K^{+} K^{-}$mode, and on the first reconstruction in hadron collisions of the suppressed decays $B^{-} \rightarrow D\left(\rightarrow K^{+} \pi^{-}\right) K^{-}$and $B^{-} \rightarrow D\left(\rightarrow K^{+} \pi^{-}\right) \pi^{-}$.

\section{References}

[1] K. Abe et al. (Belle Collaboration), Observation of B Decays to Two Kaons, Phys. Rev. Lett. 98, 181804 (2007) [hep-ex/0608049].

[2] T. Aaltonen et al. (CDF Collaboration), Observation of New Charmless Decays of Bottom Hadron, Phys. Rev. Lett. 103, 031801 (2009) [hep-ex/0812 . 4271].

[3] T. Aaltonen et al. (CDF Collaboration), Evidence for the charmless annihilation decay mode $B_{s}^{0} \rightarrow \pi^{+} \pi^{-}$, hep-ex/1111.0485, submitted to Phys. Rev. Lett.

[4] LHCb Collaboration, Charmless charged two-body decays at LHCb with 2011 data, LHCb-CONF-2011-042; A. Carbone, these proceedings.

[5] A. Ali et al., Charmless nonleptonic $B_{s}$ decays to $P P, P V$, and $V V$ final states in the perturbative $Q C D$ approach, Phys. Rev. D 76, 074018 (2007) [hep-ph/0 703162]; Y. Li et al., Branching ratio and CP asymmetry of $B_{s}^{0} \rightarrow \pi^{+} \pi^{-}$decays in the perturbative QCD approach, Phys. Rev. D 70, 034009 (2004) [hep-ph/ 0404028$].$

[6] M. Beneke and M. Neubert, $Q C D$ factorization for $B \rightarrow P P$ and $B \rightarrow P V$ decays, Nucl. Phys. B675, 333 (2003) [hep-ph/0308039].

[7] J.-F. Sun, G.-H. Zhu, and D.-S. Du, Phenomenological Analysis of Charmless Decays $B_{s} \rightarrow P P, P V$ with QCD Factorization, Phys. Rev. D 68, 054003 (2003) [hep-ph/ 0211154].

[8] H.-Y. Cheng and C.-K. Chua, QCD Factorization for Charmless Hadronic $B_{s}$ Decays Revisited, Phys. Rev. D 80, 114026 (2009) [hep-ph / 0910 . 5237]; Revisiting charmless hadronic $B_{u, d}$ decays in QCD factorization, Phys. Rev. D 80, 114008 (2009) [hep-ph/ 0909.5229$].$

[9] B. Aubert et al. (BABAR Collaboration), Improved Measurements of the Branching Fractions for $B^{0} \rightarrow \pi^{+} \pi^{-}$and $B^{0} \rightarrow K^{+} \pi^{-}$, and a Search fro $B^{0} \rightarrow K^{+} K^{-}$, Phys. Rev. D 75, 012008 (2007) [hep-ex/0608003].

[10] D. Atwood, I. Dunietz and A. Soni, Enhanced CP Violation with $B \rightarrow K D^{0}\left(\bar{D}^{0}\right)$ Modes and

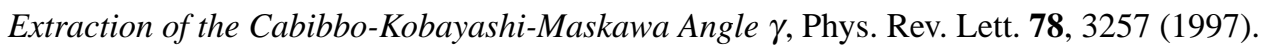


[11] T. Aaltonen et al. (CDF Collaboration), Measurements of branching fraction ratios and $C P$-asymmetries in suppressed $B^{-} \rightarrow D\left(\rightarrow K^{+} \pi^{-}\right) K^{-}$and $B^{-} \rightarrow D\left(\rightarrow K^{+} \pi^{-}\right) \pi^{-}$decays, Phys. Rev. D 84, 091504 (2011) [hep-ex/1108.5765].

[12] Y. Horii et al. (Belle Collaboration), Evidence for the Suppressed Decay $B^{-} \rightarrow D K^{-}, D \rightarrow K^{+} \pi^{-}$, Phys. Rev. Lett. 106, 231803, (2011) [hep-ex/1103.5951]; P. del Amo Sanchez et al. (BABAR Collaboration), Search for $b \rightarrow u$ transitions in $B^{-} \rightarrow D K^{-}$and $B^{-} \rightarrow D^{*} K^{-}$Decays, Phys. Rev. D 82, 072006, (2010) [hep-ex/1006.4241].

[13] LHCb Collaboration, Evidence for the suppressed decay $B^{ \pm} \rightarrow\left(K^{\mp} \pi^{ \pm}\right)_{D} K^{ \pm}$, LHCb-CONF-2011-044; G. Wilkinson, these proceedings. 